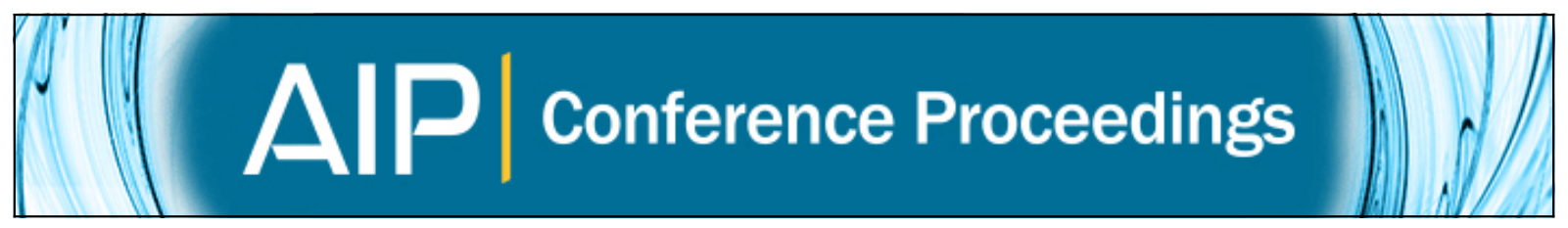

\title{
Current understanding of SEP acceleration and transport
}

C. M. S. Cohen

Citation: AIP Conference Proceedings 1720, 060001 (2016); doi: 10.1063/1.4943836

View online: http://dx.doi.org/10.1063/1.4943836

View Table of Contents: http://scitation.aip.org/content/aip/proceeding/aipcp/1720?ver=pdfcov

Published by the AIP Publishing

Articles you may be interested in

Diffusive shock acceleration and large SEP events

AIP Conf. Proc. 1539, 105 (2013); 10.1063/1.4811000

Diffusive shock acceleration in large SEP events

AIP Conf. Proc. 1436, 178 (2012); 10.1063/1.4723606

Probing SEP Acceleration Processes With Near-relativistic Electrons

AIP Conf. Proc. 1183, 3 (2009); 10.1063/1.3266783

MHD turbulence model for global simulations of the solar wind and SEP acceleration

AIP Conf. Proc. 1039, 93 (2008); 10.1063/1.2982491

Simulation of SEP Acceleration and Transport at CME-driven Shocks

AIP Conf. Proc. 781, 201 (2005); 10.1063/1.2032697 


\title{
Current Understanding of SEP Acceleration and Transport
}

\author{
C.M.S. Cohen ${ }^{1, \text { a) }}$ \\ ${ }^{1}$ California Institute of Technology, MC 290-17, Pasadena, CA USA \\ ${ }^{a)}$ Corresponding author: cohen@srl.caltech.edu
}

\begin{abstract}
Through new missions and unusual solar conditions, solar cycle 24 has afforded the opportunity for expanding our understanding of solar energetic particle (SEP) acceleration and transport. With complementary SEP observations from multiple spacecraft separated significantly in longitude, it has been possible to examine the longitudinal distribution of energetic particles in individual events, rather than relying on statistical event studies. Unprecedented $360^{\circ}$ views of the Sun, in multiple wavelengths and coronagraphs, has made it possible to identify solar source regions regardless of where they are located and to more accurately determine the properties of related coronal mass ejections. The unusually quiet conditions during the onset of cycle 24 allowed smaller SEP events to be examined and their source regions to be unambiguously identified. This paper reviews some of the unexpected results from multi-spacecraft SEP observations made over this solar cycle and discusses their implications for particle acceleration near the Sun and transport through the inner heliosphere.
\end{abstract}

\section{MULTI-SPACECRAFT MEASUREMENTS}

With its launch on 25 October 2006, the Solar Terrestrial Relations Observatory (STEREO) began a new era of space physics observations, wherein simultaneous remote and in-situ observations are routinely made from significantly different longitudinal positions, yet at nearly the same radial distances from the Sun. The STEREO mission consists of two identical spacecraft; one, STB, with a slightly longer orbital period than Earth and one, STA, with a shorter period, resulting in separations between the spacecraft and Earth that increase by $22.5^{\circ}$ per year. The instrumentation on STEREO is complementary to many of the observational packages flying on near-Earth spacecraft; e.g., the energy and species coverage for solar energetic particles (SEPs) is very similar for the ACE and STEREO spacecraft $[1,2]$ and solar imaging in EUV and white light coronagraphs are available from STEREO, SDO, and SOHO. The combination of STEREO and near-Earth measurements allows properties of SEP events to be studied as a function of longitude on an event-by-event basis. This is a significant step beyond the statistical, single-point studies that have been made in the past (e.g. [3,4]) in which the variability in the acceleration and transport conditions from one event to the next hampered interpretations of the longitudinal dependence of various SEP characteristics.

Since February 2011, when the separation between STB and STA reached $180^{\circ}$, it has been possible to view the entire surface of the Sun. This allows the source region of any SEP event to be identified and examined, whereas previously when an SEP event had no obvious source on the solar hemisphere visible from Earth, the source location could only be estimated based on information of active regions before rotating over the western limb. Sources that emerged on the 'backside' of the Sun could not be determined until days later when they rotated over the eastern limb and became visible to Earth. Clearly the lack of knowledge regarding the characteristics of these backside source regions made it impossible to fully understand the solar context in which such SEP events occurred. Worse, it was possible for the source region of an SEP event to be mis-identified based on activity occurring on the frontside of the Sun.

Unfortunately for the study of large SEP events, STEREO was launched into the quietest and longest solar minimum in decades [5,6] and no SEP events of significant size were observed until 2011. However, the quiet conditions were favorable for the study of small, ${ }^{3} \mathrm{He}$-rich events. In particular, the source regions of these events 

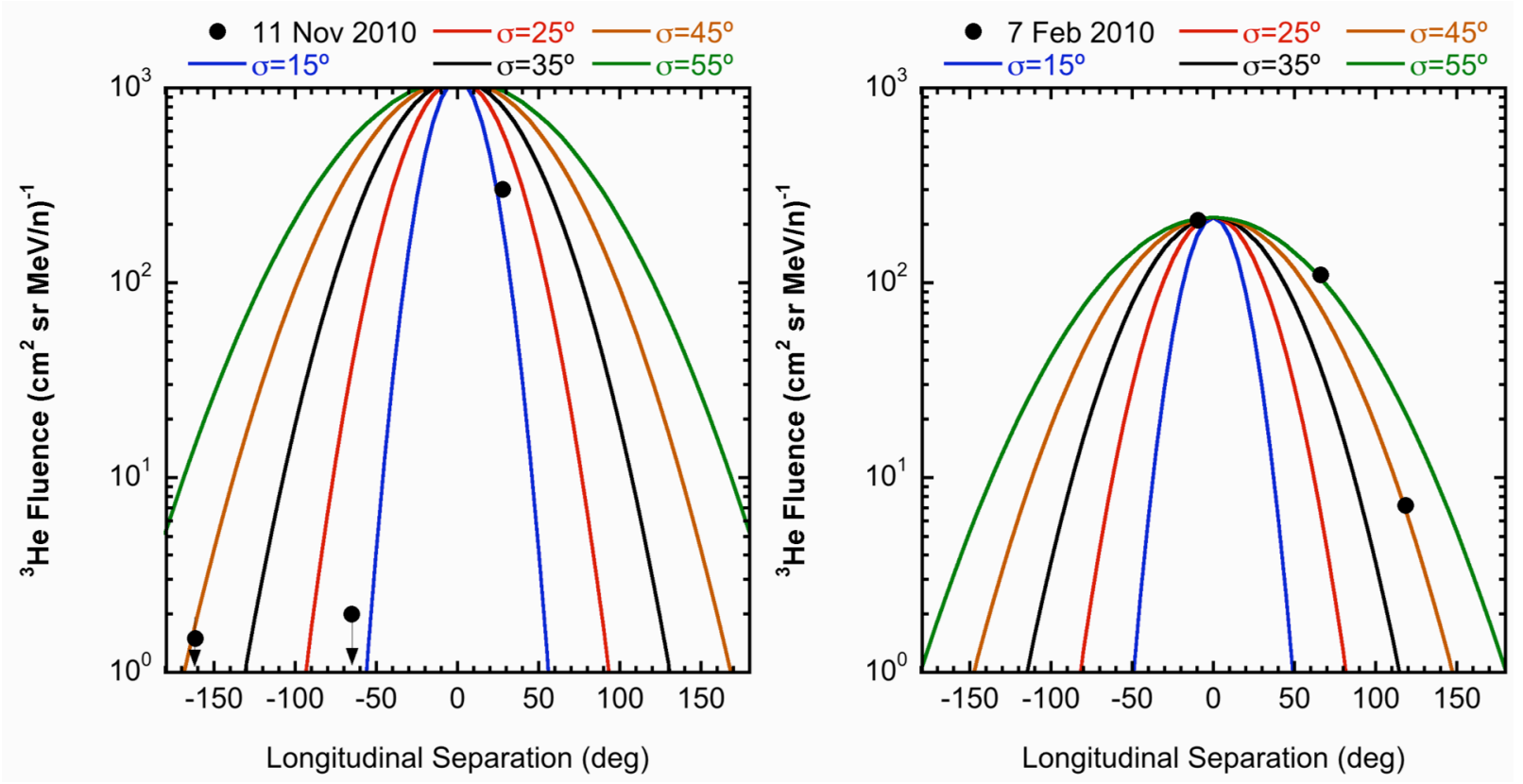

FIGURE 1. ${ }^{3} \mathrm{He}$ fluences versus the longitudinal separation between the source flare and the magnetic footpoint of the observing spacecraft. Only STB observed the 11 November 2010 event (left), STA and ACE provided upper limits to the fluence. Gaussians of different widths (as indicated above the plot), centered on the flare location, indicate that a maximum width of $\sim 15^{\circ}$ is consistent with the observations. Similar analysis for the 7 February 2010 event (with all three spacecraft measuring finite ${ }^{3} \mathrm{He}$ fluences; right) yields a longitudinal width of $\sim 45-55^{\circ}$.

were generally easily identifiable as they were often one of only a few active regions on the Sun and, in many cases, the only one actively flaring at the appropriate time (see e.g., [7]).

\section{${ }^{3}$ HE-RICH SEP EVENTS}

${ }^{3} \mathrm{He}$-rich SEP events, often referred to as 'impulsive' events because of their association with impulsive soft xray flares, exhibit several characteristics that differ substantially from larger SEP events (see [4] for a comprehensive review). The acceleration process responsible for these events is believed to be related to the magnetic reconnection processes that are occurring in the vicinity of a flare and to occur within a fairly localized region on the Sun. This is in stark contrast to the large SEP events which are created primarily through diffusive shock acceleration at interplanetary and coronal shocks driven by fast and wide coronal mass ejections (CMEs). Because of the difference in location and process of acceleration, ${ }^{3} \mathrm{He}$-rich SEP events have elemental and isotopic composition that is distinct from that of the large, shock accelerated events. In particular the ${ }^{3} \mathrm{He} /{ }^{4} \mathrm{He}$ abundance ratio can be orders of magnitude higher and the $\mathrm{Fe} / \mathrm{O}$ and $\mathrm{Ne} / \mathrm{O}$ abundance ratios can be 10 and 3 times, respectively, that seen in the larger events. These signatures make identifying such events relatively straightforward.

A study of the longitudes of the sources of ${ }^{3} \mathrm{He}$-rich SEP events measured near Earth revealed a fairly narrow distribution, centered roughly on the solar longitude typically magnetically connected with Earth via the Parker spiral and having a Gaussian sigma of $\sim 19^{\circ}$. This was consistent with the solar source being a relatively small region and indicated that such events resulted in a relatively small range of magnetic fields lines being populated with energetic particles. It was concluded that in order to see a ${ }^{3} \mathrm{He}$-rich SEP event, the observer must be fairly well connected, magnetically, to the source region [4].

Thus it was quite surprising that the 7 February $2010{ }^{3} \mathrm{He}$-rich event was observed by both STEREOs and ACE, when STB and STA were $136^{\circ}$ apart. The source region was located near central meridian (as viewed from Earth) and STB (at E71) was magnetically well connected to the region. ACE was not well connected but within $\sim 3 \sigma$ of the expected longitudinal width mentioned above, so perhaps it was not inconceivable that ACE observed the event as well. The fact that STA (at W65 and magnetically connected to a point $>130^{\circ}$ west of the source region) detected 

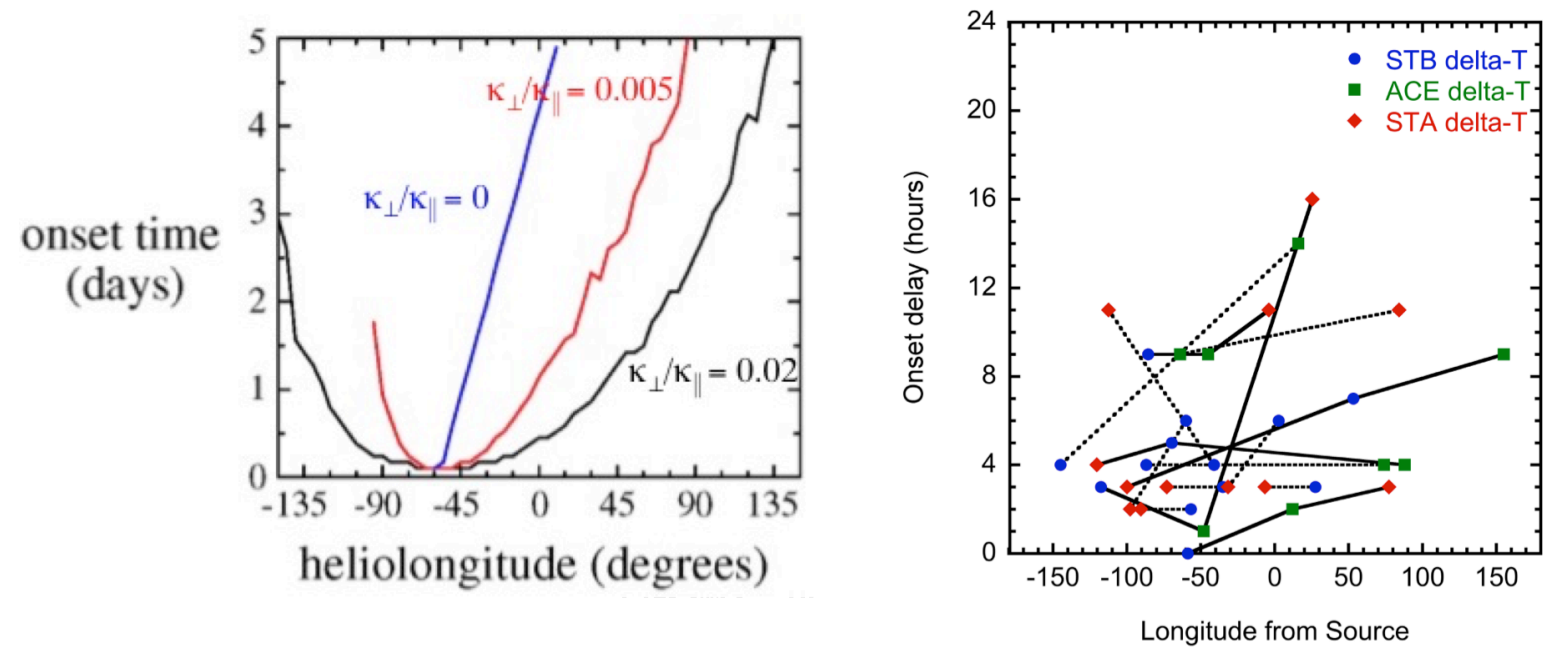

FIGURE 2. (left) Onset times for ${ }^{3} \mathrm{He}$-rich SEP events, relative to the flare time, as a function of observer longitude relative to the flare source as calculated by Giacalone and Jokipii [8] for different ratios of the perpendicular to parallel diffusion coefficients. (right) Observed delays in onsets relative to the flare time for several multi-spacecraft ${ }^{3} \mathrm{He}$-rich SEP events as a function of longitude. Note the different units for the y-axis (hours vs days); all the observed delays are significantly shorter than those predicted and the longitude dependence substantially weaker.

this event was completely at odds with expectations. A fit to the ${ }^{3} \mathrm{He}$ fluence versus longitude revealed a sigma of $48^{\circ}$, more than twice the $19^{\circ}$ derived from single-spacecraft studies. This event is discussed in detail in Wiedenbeck et al. [7] and the authors identify many other unexpectedly wide, multi-spacecraft ${ }^{3} \mathrm{He}$-rich events indicating that the 7 February 2010 event was not singular.

One of the first possibilities that presents itself when considering these events is that perhaps all ${ }^{3} \mathrm{He}$-rich events are wide, but that the instrumentation used in previous studies was not sensitive enough to determine that. This was addressed by Cohen et al. [9] by examining ${ }^{3} \mathrm{He}$-rich events with peak intensities similar to that of the 7 February 2010 event but seen by only one of the STEREO spacecraft and not by ACE (which has sensors with greater sensitivity than those on STEREO and can detect smaller events). For these single-spacecraft events, upper limits were derived for the non-observing spacecraft and Gaussian distributions of varying widths centered on the flare location were compared to the upper limits and fluence measurement versus longitude in order to determine the widest distribution consistent with the observations. An example of this analysis is given in Fig. 1 for the singlespacecraft event of 11 November 2010 as well as the same analysis technique applied to the three spacecraft measurements from the 7 February 2010 event. All the single-spacecraft events were found to have maximum widths significantly smaller than those of the wide events identified by Wiedenbeck et al., thus ruling out the possibility that all ${ }^{3} \mathrm{He}$-rich events have large longitudinal spreads.

Since not all ${ }^{3} \mathrm{He}$-rich events are wide, yet the wide events are not rare, the process(es) involved must be neither ubiquitous nor uncommon. Various suggestions have been made, of which several have been discounted: multiple flares at well separated locations has been excluded from the lack of additional flaring regions during many of the wide events [10]; magnetic field line spreading from the photosphere to $\sim 2.5 \mathrm{R}_{\mathrm{S}}$ has been shown to rarely exceed $100^{\circ}$, thus not enough to explain many of the observations [10]; and magnetic field line meandering in the interplanetary medium is expected to be of the order of only $5-10^{\circ}$ in longitude [11].

The concept that co-rotation of the magnetic field lines during wide events would carry particles westward, contributing to the observed spread, was promoted by Giacalone and Jokipii [8] after noting the significant time delay between the event onset at STA as compared to STB in the 7 February 2010 event. The authors solved the Parker transport equation while assuming different values of the particles' mean free path $(\lambda)$ and several values of the ratio of perpendicular to parallel diffusion coefficients $\left(\kappa_{\perp} / \kappa_{\|}\right)$. From these calculations the authors could predict the onset time of an SEP event at different longitudes relative to the source longitude (Fig. 2, left panel). To test this theory, Cohen et al. [12] examined the SEP onset time as a function of longitude for several multi-spacecraft ${ }^{3} \mathrm{He}-$ rich SEP events. Very few of the events showed delays as long as those seen in the 7 February 2010 event and the longitudinal dependence of the delays was minor in many of the events (Fig. 2, right panel). Overall, the delays 


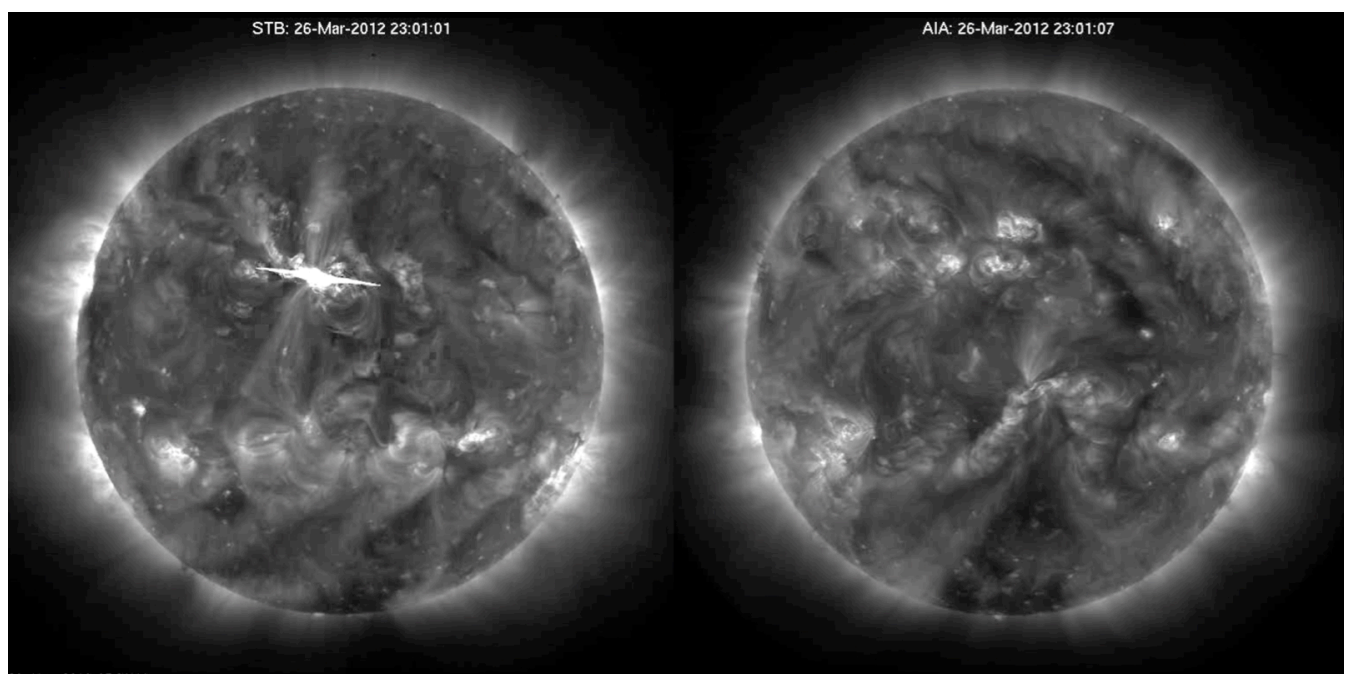

FIGURE 3. Views of the Sun in $195 \AA$ from SDO at Earth (right) and from STB (119 to the east) (left). The source of the 26 March 2012 SEP event is not evident from the Earth viewpoint but quite clear from STB.

found by Cohen et al. were significantly shorter and less dependent on longitude than predicted by the calculations of Giacalone and Jokipii. This suggests that the co-rotation of magnetic field lines is not the primary cause of the wide longitude spread observed in these ${ }^{3} \mathrm{He}$-rich events.

Other suggested spreading mechanisms show promise but have not been definitively confirmed. The proximity of coronal holes to active regions can lead to complex magnetic morphologies [13,14], which, through reconnection, can provide broad longitudinal access to particles originating from a small photospheric region [15]. Studies of the longitudinal spread and observed anisotropy of energetic electrons during multi-spacecraft SEP events suggest that interplanetary cross field diffusion and/or coronal transport may play a substantial role in particle distribution $[16,17]$. Whether this applies directly to ${ }^{3} \mathrm{He}$-rich events remains to be determined.

\section{LARGE SEP EVENTS}

Two significant events occurred in early 2011; the SEP activity began to increase with larger events being observed and the STEREO spacecraft reached $180^{\circ}$ separation allowing the full Sun to be observed simultaneously. The former meant that the longitudinal dependence of characteristics such as fluence, peak intensity, and composition could be examined for CME-driven, shock-accelerated SEP events. The latter resulted in the capability of identifying the source region for any SEP event, regardless of where on the Sun it was located, an advantage illustrated in Fig. 3. Given the traditional view available from Earth (right panel) it would be difficult to identify the source of the 26 March 2012 event; from the viewpoint of STB (left panel), the source region and associated flare are clear.

Through statistical SEP event studies made in the 1980's it was understood that the rate at which particle intensities rose and when they peaked was strongly dependent on the longitude of the observer relative to the source region [18]. For events westward of the observer, the magnetic connection to the shock front early in the event resulted in fast rises and early peaks of the particle intensities, while events east of the observer showed slow rises and peaks that occurred at or just after the shock passed the spacecraft due to the observer's relatively poor magnetic connection to the shock until late in the event. The 3 November 2011 event was subsequently quite unexpected as it was observed by both STEREOs and near-Earth spacecraft (i.e., ACE and SOHO) with similar rise and peak times even though the event occurred $155^{\circ}$ east of the Sun-Earth line and STB and STA were $102^{\circ}$ east and $105^{\circ}$ west removed from the Earth, respectively. The SEP event began at all observers within 30 minutes, indicating very efficient and fast dispersion of energetic particles throughout the inner heliosphere.

In a survey of more than $200 \mathrm{SEP}$ events with measurable $>25 \mathrm{MeV}$ protons, Richardson et al. [19] found that while not all events with sources on the backside of the Sun were observed so promptly at Earth, approximately a third of the detected events had backside sources. This underscores the importance of monitoring the full Sun for accurate predictions of SEP events and their related space weather hazards. Equally important is understanding how particles are efficiently transported over $360^{\circ}$ of longitude, currently a still elusive goal. Clues regarding the 

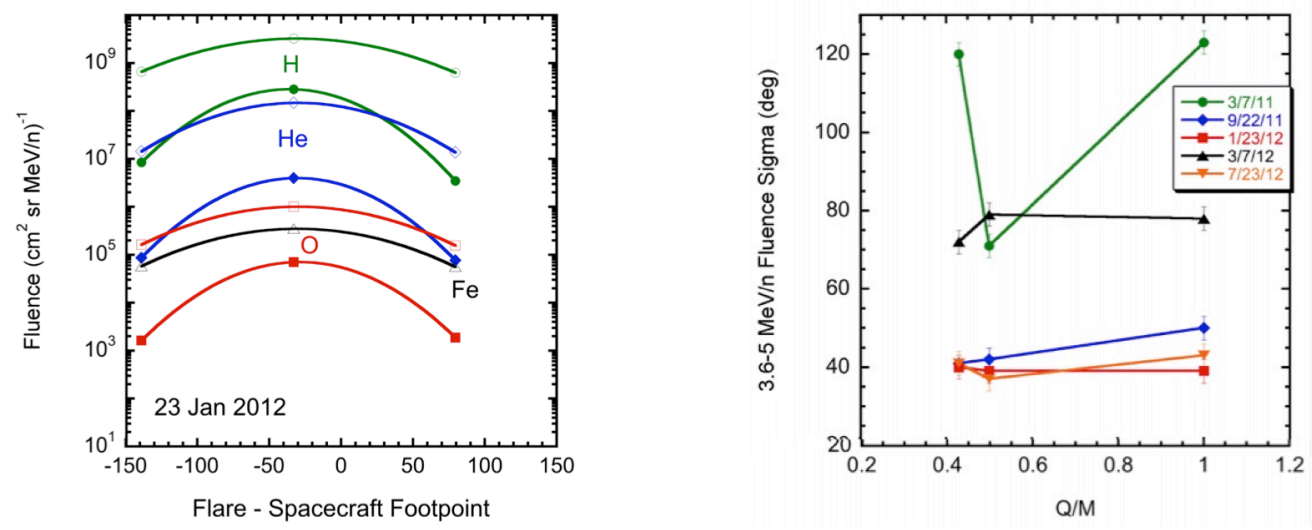

FIGURE 4. Gaussian fits to the fluences of $\mathrm{H}$ (green circles), He (blue diamonds), O (red squares), and Fe (black triangles) observed in the multi-spacecraft SEP event of 23 Jan 2012 (left). Filled symbols are for 3.5-5 MeV/nuc; open symbols are for 0.32-0.45 MeV/nuc. (right) Resulting sigmas from similar fits to the 3.6-5 MeV/nuc fluences obtained in five large SEP events.

Sigmas calculated from fluences at the lower energy interval of $0.32-0.45 \mathrm{MeV} /$ nuc similarly showed no systematic $\mathrm{Q} / \mathrm{M}$ dependence. The plot in the right panel is reprinted with permission from [20].

process(es) involved and/or the required interplanetary/coronal conditions, may be revealed through examination of different species of energetic particles. For example, rigidity-dependent processes should result in different longitudinal behaviors for elements such as $\mathrm{Fe}$, which has a lower charge-to-mass ratio $(\mathrm{Q} / \mathrm{M})$ than does $\mathrm{O}$ or $\mathrm{He}$.

The $\mathrm{Q} / \mathrm{M}$ dependence of the longitude spread of SEPs has been examined for only a handful of events. Cohen et al. [20] studied five events occurring in 2011-2012 which were observed by both STEREOs and ACE. For each event the event-integrated fluences of $\mathrm{H}, \mathrm{He}, \mathrm{O}$, and $\mathrm{Fe}$ at two different energy intervals $(0.32-0.45 \mathrm{MeV} / \mathrm{nuc}$ and 3.6-5 MeV/nuc) were calculated for each spacecraft. The magnetic footpoints of the spacecraft were determined assuming a nominal Parker spiral corresponding to $450 \mathrm{~km} / \mathrm{s}$ and the relative longitudinal distances from the source region were calculated. The $\mathrm{H}, \mathrm{He}, \mathrm{O}$, and Fe fluences vs longitude separation were fit with Gaussians for each event (see Fig. 4, left panel for an example). The Gaussian sigmas were then plotted versus Q/M values (where charge states of 7 and 15 were assumed for $\mathrm{O}$ and $\mathrm{Fe}$, respectively) to determine if a systematic $\mathrm{Q} / \mathrm{M}$ dependence was present. Fe fluences were only obtained for the lower energy interval, but there was no clear $\mathrm{Q} / \mathrm{M}$ dependence to the longitude sigmas for either energy range (Fig. 4, right panel). Two events exhibited significantly larger sigmas and it was suggested that these events were affected by additional CME activity during the course of the events which did not occur in the other three events. Although the lack of $\mathrm{Q} / \mathrm{M}$ organization suggests that rigidity is not a controlling factor in the longitudinal spread of energetic particles, it is clear that the results are highly influenced by the event-to-event variability in such a small sample size and that a larger survey-type study should be performed.

\section{SUMMARY}

Much has been learned about the distribution of energetic particles in the inner heliosphere during both small and large SEP events, largely due to the contribution of measurements made by instrumentation on the STEREO spacecraft. With these sensors, not only can SEPs be observed simultaneously at different locations during individual events, the remote sensing instruments allow the source regions anywhere on the Sun to be identified and their characteristics examined. From multi-spacecraft studies using STEREO observations several new and important results have emerged, a few of which have been highlighted here.

${ }^{3} \mathrm{He}$-rich SEP events were found to not always be confined to narrow longitudinal regions magnetically connected to the solar source. These wide events are not uncommon, but neither are they universal. The current most promising candidates for processes governing the wide distribution of SEPs from these small source regions involve complex reconnection configurations at the Sun and/or unusual transport conditions (probably involving significant cross-field diffusion) in the interplanetary medium.

The longitude distribution of particles in large SEP events can be surprisingly fast and extensive, resulting in events being observed over $360^{\circ}$ within tens of minutes. The contribution of sources out of view from the Earth to SEP events impacting the Earth cannot be ignored, thus continual remote sensing observations far removed from the Sun-Earth line is critical to accurate predictive capabilities. As with the spread of ${ }^{3} \mathrm{He}$-rich SEP events, the 
mechanism(s) responsible is still not well determined. Although one study indicates that the particle's rigidity is not a controlling factor, the very limited sample size makes it difficult to generalize this result. For large SEP events, it is likely that an extensive shock region near the Sun, combined with unusual interplanetary transport conditions, is involved, however, much work remains to be done.

The upcoming missions, Solar Probe Plus and Solar Orbiter, will provide another dimension to SEP studies. By sampling the heliosphere inside $30 \mathrm{R}_{\mathrm{S}}$, the missions will yield critical information regarding the conditions where much of the particle acceleration occurs. Of particular interest will be the characteristics of the suprathermal seed population, the interplanetary shocks, and the local turbulence. Undoubtedly, the results will yield additional surprises that will challenge our current assumptions and, hopefully, ultimately lead to a better understanding of particle acceleration and transport.

\section{ACKNOWLEDGMENTS}

The author thanks the SDO/AIA and STEREO/SECCHI team for making their movie and image data available. Additional thanks to J. Giacalone for his discussions on co-rotation, field line meandering, and particle diffusion and for the creation of the left panel of Fig 2.

\section{REFERENCES}

1. E. C. Stone, A. M. Frandsen, R. A. Mewaldt, E. R. Christian, D. Margolies, J. F. Ormes, and F. Snow, Space Science Reviews 86, 1 (1998).

2. J. G. Luhmann, et al., Space Science Reviews 136, 117 (2008).

3. H. V. Cane, R. A. Mewaldt, C. M. S. Cohen, and T. T. von Rosenvinge, Journal of Geophysical Research 111, A06S90 (2006).

4. D. V. Reames, Space Science Reviews 90, 413 (1999).

5. R. A. Mewaldt, A. J. Davis, K. A. Lave, R. A. Leske, E. C. Stone, M. E. Wiedenbeck, W. R. Binns, E. R. Christian, A. C. Cummings, G. A. de Nolfo, M. H. Israel, A. W. Labrador, and T. T. von Rosenvinge, The Astrophysical Journal Letters 723, L1 (2010).

6. D. J. McComas, N. Angold, H. A. Elliott, G. Livadiotis, N. A. Schwadron, R. M. Skoug, and C. W. Smith, The Astrophysical Journal 779, 2 (2013).

7. M. E. Wiedenbeck, G. M. Mason, C. M. S. Cohen, N. V. Nitta, R. Gómez-Herrero, and D. K. Haggerty, The Astrophysical Journal 762, 54 (2013).

8. J. Giacalone and J. R. Jokipii, The Astrophysical Journal Letters 751, L33 (2012).

9. C. M. S. Cohen, M. E. Wiedenbeck, G. M. Mason, R. Gómez-Herrero, D. K. Haggerty, and N. V. Nitta, in Proceedings of the 34th International Cosmic Ray Conference (2013), paper 0802.

10. M. Wiedenbeck, G. M. Mason, C. M. S. Cohen, cohen, N. V. Nitta, R. Gómez-Herrero, and D. K. Haggerty, in Proceedings of the 32nd International Cosmic Ray Conference (2011), paper 208.

11. J. Giacalone, (private communication).

12. C. M. S. Cohen, J. Giacalone, G. M. Mason, and M. E. Wiedenbeck, The Astrophysical Journal in preparation, (2016).

13. V. S. Titov, Z. Mikić, J. A. Linker, R. Lionello, and S. K. Antiochos, The Astrophysical Journal 731, 111 (2011).

14. S. K. Antiochos, J. A. Linker, R. Lionello, Z. Mikić, V. Titov, and T. H. Zurbuchen, Space Science Reviews 172, 169 (2012).

15. D. I. Pontin and P. F. Wyper, The Astrophysical Journal 805, 39 (2015).

16. N. Dresing, R. Gómez-Herrero, B. Heber, A. Klassen, O. Malandraki, W. Droge, and Y. Kartavykh, Astronomy and Astrophysics 567, A27 (2014).

17. N. Dresing, R. Gómez-Herrero, A. Klassen, B. Heber, Y. Kartavykh, and W. Droge, Solar Physics 281, 281 (2012).

18. H. V. Cane, D. V. Reames, and T. T. von Rosenvinge, Journal of Geophysical Research 93, 9555 (1988).

19. I. G. Richardson, T. T. von Rosenvinge, H. V. Cane, E. R. Christian, C. M. S. Cohen, A. W. Labrador, R. A. Leske, R. A. Mewaldt, M. E. Wiedenbeck, and E. C. Stone, Solar Physics 289, 3059 (2014).

20. C. M. S. Cohen, R. A. Mewaldt, and G. M. Mason, Astronomical Society of the Pacific 484, 24 (2014). 